\title{
Comparison of the Hounsfield unit values obtained from cone-beam computed tomography (CBCT) and multidetector computed tomography (MDCT) images for different bone densities
}

\author{
Atefeh Khavid $^{1 \oplus}$, Mojgan Sametzadeh ${ }^{2 \oplus}$, Mostafa Godiny ${ }^{3 * \oplus}$, Mohammad Mehdi Moarrefpour ${ }^{4 \oplus}$
}

\author{
'Department of Oral and Maxillofacial Radiology, School of Dentistry, Kermanshah University of Medical Sciences, Kermanshah, Iran \\ ${ }^{2}$ Radiology Department, Faculty of Medical Science, Jundishapour University of Medical Sciences, Ahvaz, Iran \\ ${ }^{3}$ Department of Endodontics, School of Dentistry, Kermanshah University of Medical Sciences, Kermanshah, Iran \\ ${ }^{4}$ Students Research Committee, School of Dentistry, Kermanshah University of Medical Sciences, Kermanshah, Iran \\ *Correspondence to: Mostafa Godiny (E-mail: mostafa_goodin@yahoo.com) \\ (Submitted: 02 February 2021 - Revised version received: 17 February 2021 - Accepted: 20 March 2021 - Published online: 26 April 2021)
}

\begin{abstract}
Objective In recent years, cone-beam computed tomography (CBCT) has become a key diagnostic tool in dentistry. CBCT can provide 3D images of the maxillofacial area to help dental practitioners in diagnosis and treatment, especially implant placement and treatment of pathogenic lesions. This study aimed to compare the Hounsfield unit $(\mathrm{HU})$ values obtained from $\mathrm{CBCT}$ images for bones of different densities with the corresponding HU values from multidetector CT (MDCT) images.

Methods Cube-shaped bone blocks of identical size were cut from the middle section of the cow ribs and femur area such that they had a layer of cortical bone in their buccal, lingual, and top surfaces and trabecular bone in the middle. MDCT scans were performed using a Somatom Sensation Ct Scanner. After determining HU from the results of these scans, nine suitable specimens from different ranges of HU were chosen for comparison. HU of the CBCT images was computed by the dedicated software of the CBCT machine. Finally, HU values obtained from MDCT and CBCT were compared. Data analysis was performed using SPSS version 25 at the 0.05 significance level.

Results The results showed a statistically significant difference between the mean HU from MDCT images and the mean HU from CBCT images $(P<0.05)$. For similar specimens, $C B C T$ produced higher mean $\mathrm{HU}$ values than MDCT. The Pearson correlation test detected a significant direct relationship between the $\mathrm{HU}$ values of specimens in MDCT and CBCT $(P<0.05)$.

Conclusions For the tools and software used in this study, there was no significant difference between the HU values obtained from MDCT and $\mathrm{CBCT}$, but the mean HU obtained from CBCT was higher than that from MDCT.

Keywords Hounsfield unit, Cone beam computed tomography, Multidetector computed tomography
\end{abstract}

\section{Introduction}

Diagnosis is a fundamental part of every branch of medicine and plays a critical role in choosing the treatment plan and subsequent procedures. ${ }^{1}$ Diagnostic radiographic images are commonly used to identify, diagnose, rating, and screen the diseases that affect the head and neck area or dental system. ${ }^{2,3}$ In recent years, cone-beam computed tomography (CBCT) has become an increasingly popular diagnostic tool in dentistry, as it can provide three-dimensional (3D) images of the maxillofacial region that greatly facilitate diagnosis and treatment in this field, especially implant placement and treatment of pathogenic lesions. With the introduction and advancement of CBCT, 3D imaging has turned into a practical, accessible tool for dental practitioners. Clinical applications of CBCT in the diagnosis and treatment procedures involving oral and maxillofacial areas are increasing by the day. One of the most important treatment methods in modern dentistry is implant placement. However, the success of this treatment largely depends on the quality and quantity of bone in the implant recipient site. Research has shown that the implants inserted in bones of poor or low quantity are more likely to fail, which is why it is critical to carefully assess the bone structure before implant placement. ${ }^{1,2}$ With the emergence of new imaging techniques such as multidetector computed tomography (MDCT), CBCT, dual-energy $\mathrm{X}$-ray absorptiometry (DXA), and digital subtraction, it is now easier to measure bone mineral density (BMD) for such purposes. ${ }^{3-5}$ Among these methods, CBCT is increasingly used in dentistry for 3D imaging of oral and maxillofacial regions. ${ }^{6-8}$ The main advantages of CBCT include high accessibility, ease of use, and the ability to provide real-size data and cross-sectional, multiplan, and $3 \mathrm{D}$ reconstructions of the area of interest. ${ }^{9-11} \mathrm{CBCT}$ can provide a clear view of the location of impacted teeth, their relationship with, and effects on other teeth. ${ }^{11}$ The data obtained from CBCT can be reconstructed to illustrate incisions in the axial, coronal, and sagittal planes.

CBCT also allows the surgeon to reconstruct 3D images of the target area. ${ }^{12}$ Some researchers have argued that CBCT voxel numbers can be used to estimate the Hounsfield unit (HU) and BMD. ${ }^{13},{ }^{14}$, but many others believe that CBCT does not offer a reliable assessment of BMD. ${ }^{15,16}$ The $\mathrm{HU}$ is one of the main quantitative measures of bone density in MDCT. Finding a relationship between the $\mathrm{HU}$ of identical specimens in CBCT and MDCT could indicate the accuracy and validity of this unit for CBCT. It may open up new avenues for research on this subject. While many new CBCT machines offer a built-in tissue density estimation feature, only a few studies have evaluated the accuracy of these estimates. Therefore, the authors decided to investigate this issue. For this purpose, a comparison was made between the $\mathrm{HU}$ values obtained for a series of specimens with different bone densities from CBCT and MDCT images. 


\section{Method}

This study was carried out with the approval of the Ethics Committee (IR.KUMS.REC.1399.322) of Kermanshah University of Medical Sciences and the relevant officials of the School of Dentistry at this university. The specimens were made from the bone of a slaughtered cow with due attention to measurement accuracy. The pilot study was performed with three dry mandibles. MDCT and CBCT scanned the specimens, and their $\mathrm{HU}$ in the same anterior and posterior regions were determined. It was observed that the $\mathrm{HU}$ values obtained from CBCT images were very close to those of MDCT images (Fig. 1a, 1b). To create the specimens, bone blocks of the same size were cut from the middle section of a cow's ribs and femur area. The blocks were cube-shaped with right angles and identical dimensions and were cut such that they had a layer of cortical bone in their buccal, lingual, and top surfaces and trabecular bone in the middle. According to previous studies, ${ }^{27}$ cow bone was chosen mainly; a cow's femur bone has a density of D1, and different parts of its rib bone have a density of D2 to D4. Other reasons for choosing cow bone included easier access and the possibility of repeated imaging without significant ethical limitations. At all stages of the study, the specimens were placed in a refrigerator with a humid environment to prevent drying, which would change the tissue density (this is why dry mandibles were not used). Also, the delay between specimen preparation and final imaging was minimized to minimize density change during this period. MDCT scans were obtained using a Somatom Sensation CT Scanner. After determining the bone density of all specimens

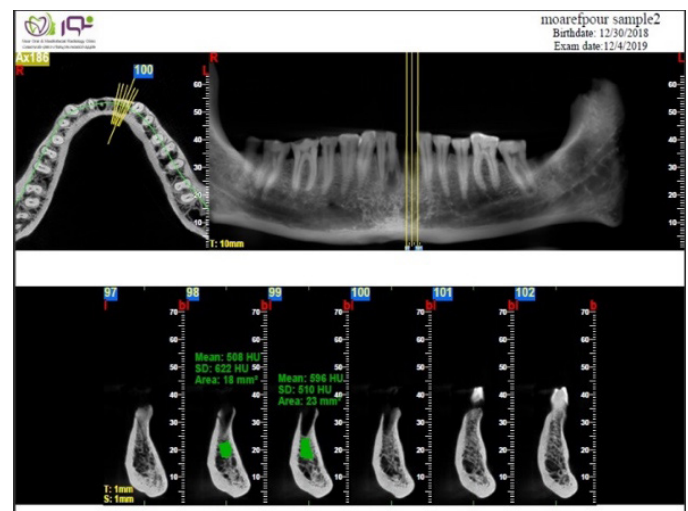

Fig. 1 (a) An example of CBCT images.
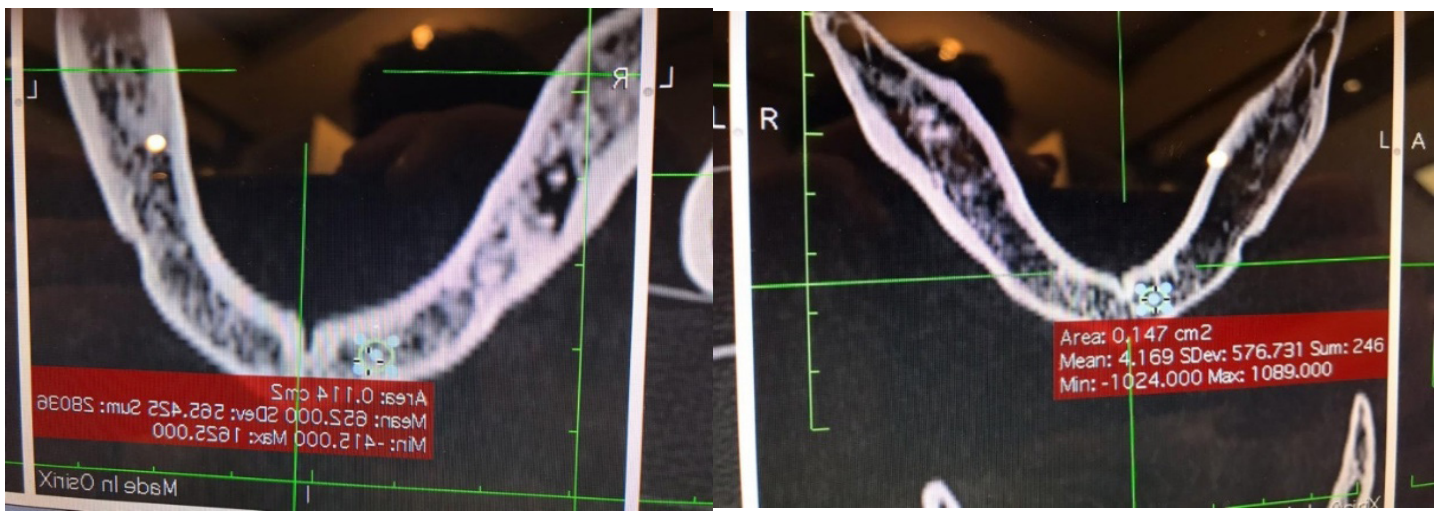

Fig. 1 (b) An example of CBCT images.

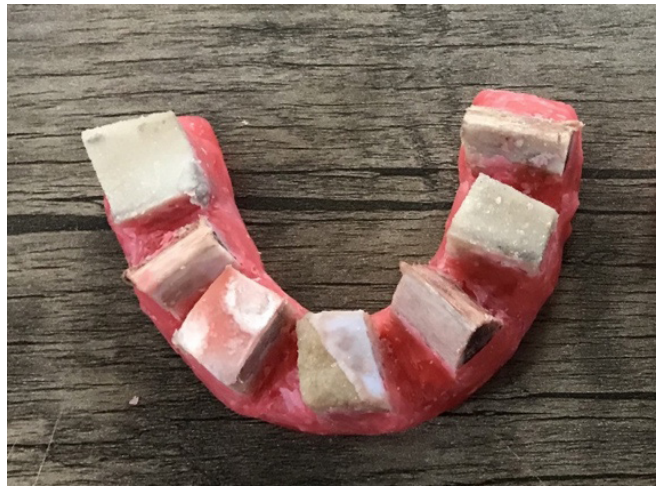

Fig. 1 (c) Specimens fixed in the wax model of the mandible. 
in $\mathrm{HU}$, nine suitable specimens from each range of $\mathrm{HU}$ were selected for use in subsequent steps. The selected specimens were numbered and then fixed on the wax model of the mandible in random order (Fig. 1c).

After taking another MDCT scan from the fixed specimens, CBCT scans were performed using a NewTom GIANO CBCT machine. In all of these scans, tissue density was obtained from the machine's dedicated software, which an expert operated. In the end, the results obtained from CBCT and MDCT were compared with each other.

A total of 52 specimens were scanned by each imaging method (104 specimens in total). The required data, which included the HU values obtained from CBCT images and those from MDCT images, were collected with a checklist. Data analysis was performed in SPSS version 25 using both descriptive and inferential statistical methods at the 0.05 significance level. The normality of data distribution was checked with the Kolmogorov-Smirnov test. Descriptive statistics (mean, standard deviation) were used to summarize and describe the quantitative characteristics of the variables. The Pearson correlation test and independent $t$-test were used to test the hypotheses.

\section{Results}

In this study, the tissue density of 52 cow bone specimens was determined by MDCT and CBCT determined the tissue density of another 52 specimens. The mean HU (tissue density) of the specimens in MDCT, and CBCT were 805.9 \pm 591.8 and 1084.5 \pm 772.6 , respectively (Table 1 ).

After establishing the normality of the data, the independent $t$-test was performed to detect differences between the means of HU values. This test showed a statistically significant difference between the mean HU in MDCT images, 805.9, and

Table 1. The mean and standard deviation of HU (tissue density) of bone specimens in MDCT and CBCT.

\begin{tabular}{lcccc}
\hline Variable & Mean & $\begin{array}{c}\text { Standard } \\
\text { deviation }\end{array}$ & Min & Max \\
\hline HU of specimens in MDCT & 805.9 & 591.8 & 16 & 1792 \\
HU of specimens in CBCT & 1084.5 & 772.6 & 134 & 2346 \\
\hline
\end{tabular}

the mean HU in CBCT images, $1084.5(\mathrm{P}<0.05)$. As can be seen, for similar specimens, CBCT provided a higher mean of HU than MDCT (Table 2). The Pearson correlation test found a significant direct relationship with a correlation coefficient of $0.978(\mathrm{P}<0.01)$ between the HU values of the specimens in MDCT and those of the specimens in CBCT (Fig. 2). As shown in Table 3, the Chi-square test detected no statistically significant difference between the $\mathrm{HU}$ values obtained from MDCT and CBCT images $(\mathrm{P}<0.05)$, suggesting that the two imaging methods offer similar results in terms of HU.

\section{Discussion}

Bone density assessments are essential for deciding when implant placement is a viable treatment and for the ultimate success of this procedure. Therefore, these assessments need to be made based on reasonably reliable imaging tests. Thus, there have been many studies on the estimation of bone density with different methods. ${ }^{17}$ Initially, these assessments were being

Table 2. Comparison of mean HU of the specimens in MDCT and $\mathrm{CBCT}$ images.

\begin{tabular}{lccccc}
\hline \multirow{2}{*}{ Variable } & MDCT & \multicolumn{1}{c}{ CBCT } & & \\
\cline { 2 - 3 } & $\begin{array}{c}\text { Mean } \pm \\
\text { standard } \\
\text { deviation }\end{array}$ & $\begin{array}{c}\text { Mean } \pm \\
\text { standard } \\
\text { deviation }\end{array}$ & t & P-value \\
\hline $\begin{array}{l}\text { HU of } \\
\text { specimens }\end{array}$ & $805.9 \pm 591.8$ & $1084.5 \pm 772.6$ & -2.064 & 0.042 \\
\hline
\end{tabular}

Table 3. Relative and absolute frequency distribution of $\mathrm{MU}$ of the specimens from MDCT and CBCT images.

\begin{tabular}{lcccc}
\hline MU / Group & MDCT & \multicolumn{1}{c}{ CBCT } & & Chi- \\
\cline { 2 - 3 } & $\begin{array}{c}\text { Frequency } \\
\text { (percentage) }\end{array}$ & $\begin{array}{c}\text { Frequency } \\
\text { (percentage) }\end{array}$ & $\begin{array}{c}\text { square } \\
\text { P-value }\end{array}$ & \\
\hline D1 $<\mathbf{1 2 5 0}$ & $19(50)$ & $19(50)$ & & \\
$\mathbf{8 5 0 - 1 2 5 0}$ & $7(50)$ & $7(50)$ & 6.47 & 0.091 \\
$\mathbf{3 5 0 - 8 5 0}$ & $6(28.6)$ & $15(71.4)$ & & \\
$\mathbf{0 - 3 5 0}$ & $20(64.5)$ & $11(35.5)$ & & \\
\hline
\end{tabular}

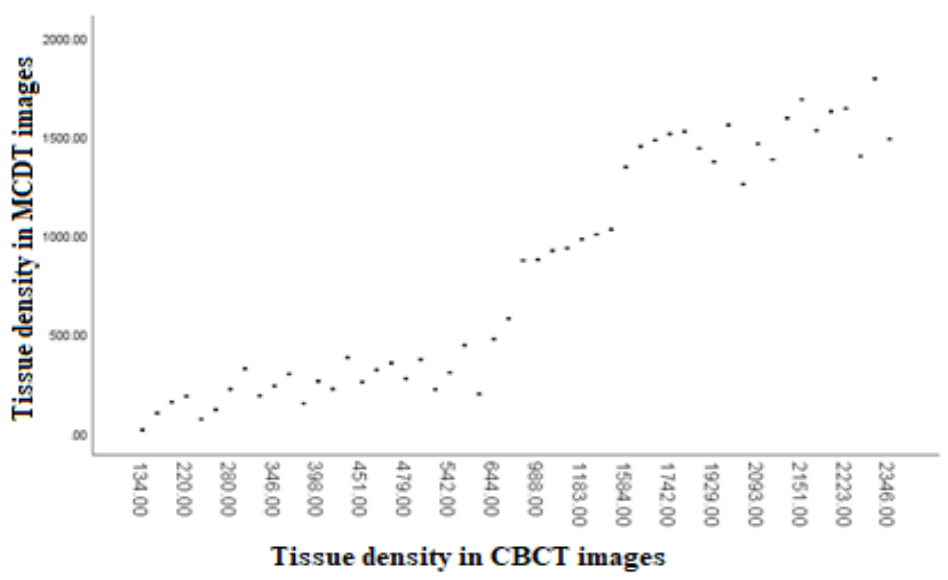

Fig. 2 Scatter plot of MU of the specimens from MDCT and CBCT images. 
performed theoretically, but eventually, the HU was introduced to standardize the relationship between theoretical assessments and bone density. Research has shown that high bone density is correlated with the success of implants. Also, high density is associated with the good initial stability of the implant. Recently, CBCT has replaced MDCT in many areas of the dental practice and is increasingly used for pre-implant imaging, thanks to its ability to capture mineralized tissues. Examining the $\mathrm{HU}$ values obtained for identical specimens from two different imaging techniques can shed more light on the accuracy and the differences of the two methods, which can have important implications for the diagnosis and the subsequent treatments.

In this study, the findings showed no significant difference between the two imaging methods in terms of the obtained MU values. However, the mean $\mathrm{HU}$ in $\mathrm{CBCT}$ images was higher than the mean HU in MDCT. This finding is consistent with the results of a study by Sliva et al. ${ }^{18}$, in which, after examining CBCT and MDCT images taken from 20 dried mandibles, the mean HU obtained from CBCT images was significantly higher. In a study by Nackaerts et al., different CBCT machines reported different $\mathrm{HU}$ values for the same specimens, but this was not an issue in MDCT. ${ }^{15}$ After comparing the $\mathrm{HU}$ and Gray values obtained with 11 different CBCT machines for the same specimens, Mah et al. ${ }^{19}$ also reported differences in the densities estimated by these machines. However, other studies such as Nomura et al. ${ }^{14}$ have obtained similar voxel values from CBCT and MDCT, indicating that CBCT could still be a good imaging method for face assessments. The discrepancies mentioned above could be due to various reasons, including the low accuracy of the software. These differences may also be attributed to the cone angle of the beams, which is the main difference between $\mathrm{CBCT}$ and MDCT imaging techniques, because as the region of interest gets larger and the said angle increases, so does the inaccuracy of measurements. According to Nackaerts et al., ${ }^{15}$ tissue density estimates obtained from CBCT are unreliable because they vary with the machine, imaging parameters, and imaged area. This issue could be extremely important for implant placement because an error in the reported HU may result in the misidentification of the bone type (D1, D2, D3, D4), which may put the operation at risk. The most important limitation of this study was the inability to use live specimens because of high X-ray exposure.

\section{Conclusion}

Considering the tools and software used in this study, there was no statistically significant difference between the HU values obtained from MDCT and CBCT images. Still, the mean HU obtained from CBCT was higher than MDCT. It is recommended to design and perform a similar study to investigate the role of the software error in the observed differences.

\section{References}

1. Payahoo S, Jabbari G. The ability of cone beam computed tomography to predict osteopenia and osteoporosis via radiographic density derived from cervical vertebrae. Int J Scient Res Dental Med Sci. 2019;1(2):18-22. 10.30485/ijsrdms.2019.89758.

2. Resnik RR, Kircos LT, Misch CE. Diagnostic imaging and techniques. Contemporary Implant Dentistry. Missouri: Mosby. 2007:38-67.

3. Isoda K, Ayukawa Y, Tsukiyama Y, Sogo M, Matsushita Y, Koyano K. Relationship between the bone density estimated by cone-beam computed tomography and the primary stability of dental implants. Clini Oral Implants Res. 2012;23(7):832-6. https://doi.org/10.1111/j.16000501.2011.02203.x.

4. González-García R, Monje F. The reliability of cone-beam computed tomography to assess bone density at dental implant recipient sites: a histomorphometric analysis by micro-CT. Clin Oral Implants Res. 2013;24(8):871-9. https://doi.org/10.1111/j.1600-0501.2011.02390.x.

5. Fuster-Torres MÁ, Peñarrocha-Diago M, Peñarrocha-Oltra D, PeñarrochaDiago M. Relationships between bone density values from cone beam computed tomography, maximum insertion torque, and resonance frequency analysis at implant placement: a pilot study. Int J Oral Maxillofac Implants. 2011;26(5): 1051-56.

6. Yabroudi F, Sindet-Pedersen S. Cone beam tomography (CBCT) as a diagnostic tool to assess the relationship between the inferior alveolar nerve and roots of mandibular wisdom teeth. Smile Dent J. 2012;7(3):12-6.

7. Feldkamp LA, Davis LC, Kress JW. Practical cone-beam algorithm. Josa A. 1984;1(6):612-9. https://doi.org/10.1364/JOSAA.1.000612.

8. Aponte Mendez M, Kayasöken G, Afjeh Soleymani B, Ravanbakhsh B. Evaluation outcome of cone beam computed tomography for treatment plan success and failure: a systematic review. Int I Scient Res Dental Med Sci. 2020;2(2):46-51. 10.30485/IJSRDMS.2020.233140.1061

9. Kipp DP, Goldstein BH, Weiss WW. Dysesthesia after mandibular third molar surgery: a retrospective study and analysis of 1,377 surgical procedures. J Am Dental Assoc. 1980;100(2):185-92. https://doi.org/10.14219/jada. archive.1980.0074

10. Libersa P, Savignat M, Tonnel A. Neurosensory disturbances of the inferior alveolar nerve: a retrospective study of complaints in a 10-year period.

J Oral Maxillofac Surg. 2007;65(8):1486-9. https://doi.org/10.1016/j. joms.2007.03.023.

11. Monaco G, Montevecchi M, Bonetti GA, Gatto MR, Checchi L. Reliability of panoramic radiography in evaluating the topographic relationship between the mandibular canal and impacted third molars. J Am Dental Assoc. 2004;135(3):312-8. https://doi.org/10.14219/jada.archive.2004.0179.

12. Deepak C, Saravanan B, Kumar SK. CBCT-A Paradigm shift in the management of dental impactions. Ind J Multidisc Dent. 2011;1(2).

13. Naitoh M, Hirukawa A, Katsumata A, Ariji E. Evaluation of voxel values in mandibular cancellous bone: relationship between cone-beam computed tomography and multislice helical computed tomography. Clin Oral Implants Res. 2009;20(5):503-6. https://doi.org/10.1111/j.1600-0501.2008.01672.x.

14. Nomura Y, Watanabe H, Honda E, Kurabayashi T. Reliability of voxel values from cone-beam computed tomography for dental use in evaluating bone mineral density. Clin Oral Implants Res. 2010;21(5):558-62. https://doi. org/10.1111/j.1600-0501.2009.01896.x.

15. Nackaerts $O$, Maes F, Yan H, Couto Souza P, Pauwels R, Jacobs R. Analysis of intensity variability in multislice and cone beam computed tomography. Clin Oral Implants Res. 2011;22(8):873-9. https://doi.org/10.1111/j.16000501.2010.02076.x.

16. Hua Y, Nackaerts O, Duyck J, Maes F, Jacobs R. Bone quality assessment based on cone beam computed tomography imaging. Clin Oral Implants Res. 2009;20(8):767-71. https://doi.org/10.1111/j.1600-0501.2008.01677.x.

17. Arisan V, Karabuda ZC, Avsever H, Özdemir T. Conventional multi-slice computed tomography (CT) and cone-beam CT (CBCT) for computerassisted implant placement. Part I: Relationship of radiographic gray density and implant stability. Clin Implant Dent Related Res. 2013;15(6):893-906. https://doi.org/10.1111/j.1708-8208.2011.00436.x.

18. Silva IM, Freitas DQ, Ambrosano GM, Bóscolo FN, Almeida SM. Bone density: comparative evaluation of Hounsfield units in multislice and cone-beam computed tomography. Braz Oral Res. 2012;26(6):550-6. https://doi. org/10.1590/S1806-83242012000600011.

19. Mah P, Reeves TE, McDavid WD. Deriving Hounsfield units using grey levels in cone beam computed tomography. Dentomaxillofac Radiol. 2010;39(6):323-35. 